\title{
Article \\ Systematic Analysis and Biochemical Characterization of the Caffeoyl Shikimate Esterase Gene Family in Poplar
}

\author{
Xuechun Wang ${ }^{1,2,3,4,+}$, Nan Chao ${ }^{1,5,+}$, Aijing Zhang ${ }^{1,2,3,4} \mathbb{( D}$, Jiaqi Kang ${ }^{1,2,3,4} \mathbb{D}$, Xiangning Jiang ${ }^{1,2,3,4}$ \\ and Ying Gai $1,2,3,4, *$ (D)
}

1 College of Biological Sciences and Biotechnology, Beijing Forestry University, Beijing 100083, China; wangxuechun0717@126.com (X.W.); chaonan1989@126.com (N.C.); zhangaijing217@163.com (A.Z.); Kangjiaqi1998@163.com (J.K.); jiangxn@bjfu.edu.cn (X.J.)

2 The Tree and Ornamental Plant Breeding and Biotechnology Laboratory, National Forestry and Grassland Administration, Beijing 100083, China

3 National Engineering Laboratory for Tree Breeding, Beijing 100083, China

4 Key Laboratory of Genetics and Breeding in Forest Trees and Ornamental Plants, Ministry of Education, Beijing 100083, China

5 Jiangsu Key Laboratory of Sericutural Biology and Biotechnology, School of Biotechnology, Jiangsu University of Science and Technology, Zhenjiang 212018, China

* Correspondence: gaiying@bjfu.edu.cn; Tel.: +86-10-6233-8063

+ These authors contributed equally to this work.

Citation: Wang, X.; Chao, N.; Zhang,

A.; Kang, J.; Jiang, X.; Gai, Y.

Systematic Analysis and Biochemical Characterization of the Caffeoyl Shikimate Esterase Gene Family in Poplar. Int. J. Mol. Sci. 2021, 22, 13366 https://doi.org/10.3390/

ijms222413366

Academic Editor: Frank M. You

Received: 2 November 2021

Accepted: 10 December 2021

Published: 13 December 2021

Publisher's Note: MDPI stays neutral with regard to jurisdictional claims in published maps and institutional affiliations.

Copyright: (c) 2021 by the authors. Licensee MDPI, Basel, Switzerland. This article is an open access article distributed under the terms and conditions of the Creative Commons Attribution (CC BY) license (https:/ / creativecommons.org/licenses/by/ $4.0 /)$.

\begin{abstract}
Caffeoyl shikimate esterase (CSE) hydrolyzes caffeoyl shikimate into caffeate and shikimate in the phenylpropanoid pathway. In this study, we performed a systematic analysis of the CSE gene family and investigated the possible roles of CSE and CSE-like genes in Populus. We conducted a genome-wide analysis of the CSE gene family, including functional and phylogenetic analyses of CSE and CSE-like genes, using the poplar (Populus trichocarpa) genome. Eighteen CSE and CSE-like genes were identified in the Populus genome, and five phylogenetic groups were identified from phylogenetic analysis. CSEs in Group Ia, which were proposed as bona fide CSEs, have probably been lost in most monocots except Oryza sativa. Primary functional classification showed that PoptrCSE1 and PoptrCSE2 had putative function in lignin biosynthesis. In addition, PoptrCSE2, along with PoptrCSE12, might also respond to stress with a function in cell wall biosynthesis. Enzymatic assay of PoptoCSE1 (Populus tomentosa), -2 and -12 showed that PoptoCSE1 and -2 maintained CSE activity. PoptoCSE1 and 2 had similar biochemical properties, tissue expression patterns and subcellular localization. Most of the PoptrCSE-like genes are homologs of AtMAGL (monoacylglycerol lipase) genes in Arabidopsis and may function as MAG lipase in poplar. Our study provides a systematic understanding of this novel gene family and suggests the function of CSE in monolignol biosynthesis in Populus.
\end{abstract}

Keywords: caffeoyl shikimate esterase; enzymatic assay; gene family; lignin; Populus

\section{Introduction}

Lignin is one of the most abundant biomass components in plants and has been extensively studied for decades because of its vital role in the formation of the plant secondary cell wall and wood [1-4]. The recalcitrance of lignin restricts both forage utilization and processing of biomass for biofuels [5,6]. Therefore, genetic modification strategies targeting key genes involved in monolignol biosynthesis are being developed to obtain plants with less lignin, or with more easily degradable lignin, while maintaining normal biomass [7-12].

The monolignol biosynthesis pathway is a branch of the phenylpropanoid pathway and has been continuously revised in recent decades. Eleven enzyme families and twentyfour metabolites have been reported to participate in monolignol biosynthesis [13]. The general phenylpropanoid pathway contains phenylalanine ammonia-lyase (PAL), cinnamate 
4-hydroxylase $(\mathrm{C} 4 \mathrm{H})$ and 4-coumarate: $\mathrm{CoA}$ ligase $(4 \mathrm{CL})$ and provides hydroxycinnamoylCoA esters as precursors for a wide range of end products. These hydroxycinnamoyl-CoA esters are further catalyzed into monolignols by a series of enzymes in the specific monolignol biosynthesis pathway [14]. Changing the mass flux in the phenylpropanoid pathway has become a strategy for modifying lignin content [2,5,15-18]. Recently, dynamic flux modeling for monolignol biosynthesis was also proposed based on systematic understanding of the monolignol biosynthesis pathway [19-22]. The discovery of caffeoyl shikimate esterase (CSE) as a central enzyme in the lignin biosynthetic pathway promoted a revision of the pathway [18]. Arabidopsis thaliana cse mutants have shown that lignin content declines with increasing levels of $p$-hydroxyphenyl units, and the efficiency of the conversion of cellulose to glucose in cse mutants is improved after saccharification without pretreatment. CSE together with $4 \mathrm{CL}$ directs the flux away from $\mathrm{H}$ lignin and toward $\mathrm{G}$ and $\mathrm{S}$ lignin. Down-regulation of CSE in hybrid poplar (Populus tremula $\times$ Populus alba) resulted in up to $25 \%$ reduced lignin deposition and increased levels of $p$-hydroxyphenyl units in the lignin polymer and cellulose content [23].

AtCSE (At1g52760), reported as LysoPL2 (Lysophospholipase 2), can be combined with acyl-CoA-binding protein 2 (ACBP2) and lysophosphatidylcholine (lysoPC) to promote the tolerance of Arabidopsis to cadmium-induced oxidative stress [24]. Vanholme et al. (2013) reported that caffeoyl shikimate esterase in Arabidopsis, AtCSE, had the ability to convert caffeoyl shikimate into caffeate [23]. Both in vitro enzymatic analyses and genetic cse mutants confirmed its vital function in monolignol biosynthesis in Arabidopsis. Although CSE orthologs were firstly reported based on phylogenetic analysis in several plants such as poplar, it is confused that protein extracts from secondary differentiating xylem of Populus trichocarpa showed no detectable CSE activity; similar results were also reported in Panicum virgatum and Oryza sativa [25]. In a recent study, detectable CSE activity was reported in crude extraction of $P$. virgatum and purified recombinant CSE proteins from Medicago truncatula and Populus deltoides [26]. The authors also indicated that reaction catalyzed by CSE may not be essential for lignification in all plant species since they found that crude protein extracts from stems of Brachypodium distachyon and Zea mays, which have no orthologs of the currently characterized CSE genes, exhibit only a weak esterase activity with caffeoyl shikimate [26]. In addition, genuine CSE was also characterized in Pinus in our previous study [27]. Two bona fide CSEs were characterized in hybrid poplar (P. tremula $\times$ P. alba), and down-regulation of CSE in hybrid poplar resulted in the accumulation of feruloyl shikimate and sinapoyl shikimate, indicating that these compounds also are in vivo CSE substrates [23]. In a recent study, the lignin of cse1 and cse2 single mutants in hybrid poplar (P. tremula $\times$ P. alba) by CRISPR-Cas9 had the same levels as the wild type, but both single mutants accumulated caffeoyl shikimate, whereas there was a 35\% reduction in lignin in the cse1 cse2 double mutant [28]. However, Jang et al. (2021) found that the lignin deposition of cse1 and cse 2 mutants in P. alba $\times$ Populus glandulosa by CRISPR-Cas9 was reduced by $29.1 \%$, although $\operatorname{cse} 1$ and $\operatorname{cse} 2$ were indistinguishable from the wild type in morphology and showed no significant difference in growth [29].

It is necessary to consider all key genes and their homologs involved in monolignol biosynthesis when establishing a dynamic flux model based on enzymatic activity since both the functional redundant and complementation homologs would affect the flux model $[19,25]$. Therefore, using the poplar (P. trichocarpa) genome, which has complete genome information and annotation, we performed a genome-wide analysis of the CSE gene family. Based on the analysis of promoter and expression profiles, we propose the functions of these genes. Biochemical characterization of the putative bona fide CSE homologs from Populus tomentosa was also performed in vitro to confirm their catalytic function. Our study provides a further understanding of this novel gene family and new target genes for modifying the phenylpropanoid pathway. 


\section{Results}

\subsection{Organization and Distribution of the CSE Gene Family}

We found that the number of CSE gene family members differed among the examined species, and the CSE gene family may have experienced duplication or loss. Eighteen CSE homologs were identified in poplar. These PoptrCSE or PoptrCSE-like genes are distributed on 13 chromosomes (Figure 1), indicating that block duplication resulting from 'Salicoid' genome duplication may play a role in the formation of the CSE family [30]. Four chromosomes, including chromosomes I, VIII, X and XI, contain two or three PoptrCSE genes, most of which are located in homologous duplicated blocks and form ten duplicated pairs. PoptrCSE3 is located on a duplicated segment on chromosome XI without a matching duplicate. It is possible that its duplicate was lost during evolution. Ks analysis of the duplicated CSE gene pairs showed that 7 of the 10 CSE gene pairs experienced a recent duplication event, with Ks values between 0.2 and 0.4 (Supplementary Table S1).

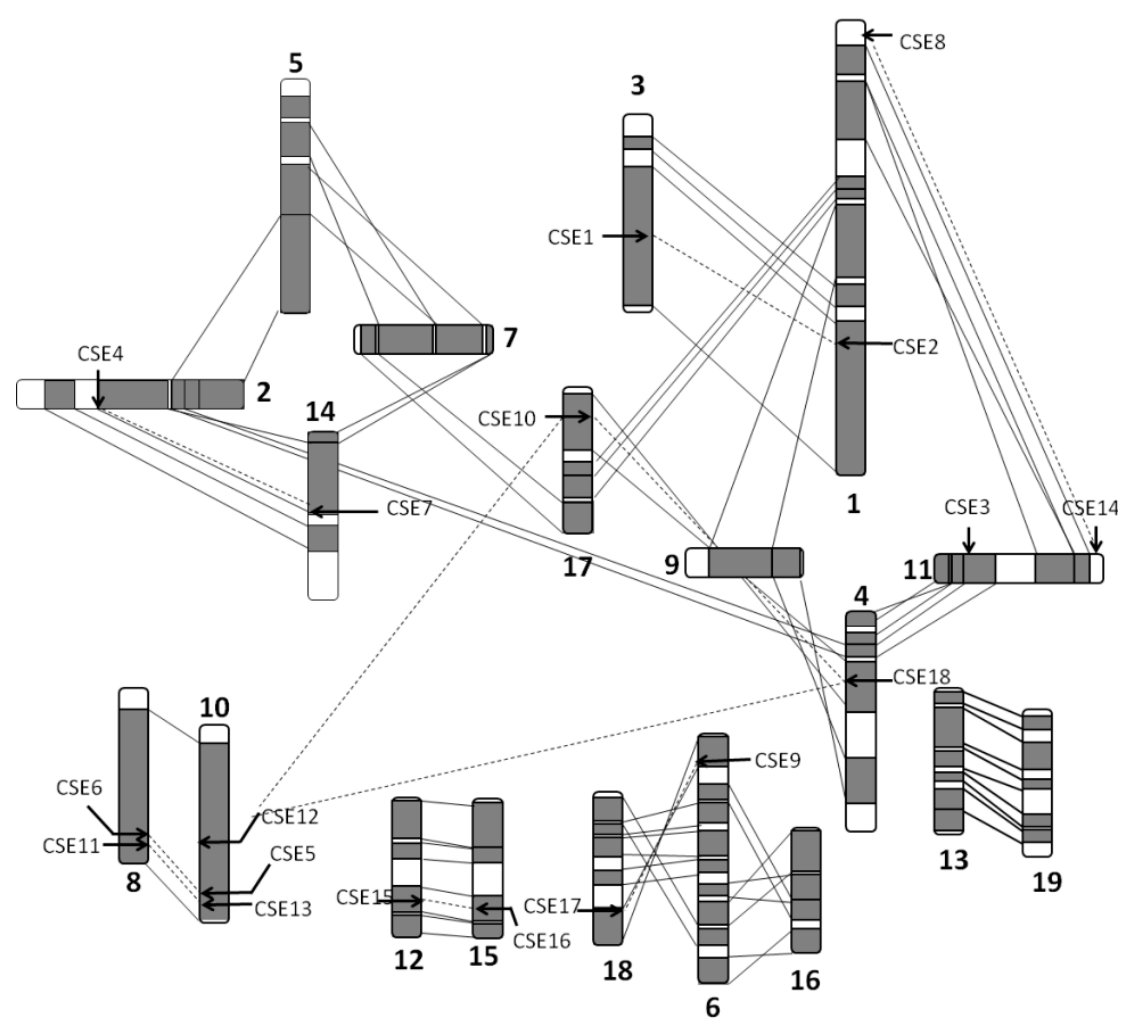

Figure 1. Distribution of CSE and CSE-like genes on Populus chromosomes. Regions that are assumed to correspond to homologous genome blocks are shaded gray and connected by lines. Schematic view of chromosome reorganization from the most recent whole-genome duplication in Populus was adapted from Tuskan et al. (2006), based on duplication coordinates from the genome assembly version 3.0. The position of genes is indicated by an arrowhead, and positional paralogs relative to the duplicated blocks are connected by dashed lines. The numbers of 1-18 are chromosome numbers.

The CSE gene family was also screened from a number of plant species, including mosses (Physcomitrella patens), ferns (Selaginella moellendorffii), gymnosperms (Picea sitchensis) and angiosperms, in the present study. Eighteen PoptrCSE and PoptrCSE-like genes were identified in P. trichocarpa. These PoptrCSE genes were distributed on 13 chromosomes with homologous pairs, except for PoptrCSE3, which was also located in a duplicate block. Inter-species collinear analysis revealed that PoptrCSE3 is orthologous to the AtrCSE1 gene in Amborella, which is the most primitive branch of angiosperms, while we could not find its positional paralog relative to the duplicated blocks in Populus (Figure 2b). In addition, based on the aligned gene strings of a set of homologous segments with PoptrCSE11 and PoptrCSE13, and the fact that PoptrCSE13 only has a $361 \mathrm{bp}$ upstream sequence, we propose 
that it is an insertion of a new gene (PT10G22220) into the promoter region of PoptrCSE13 that leads to this change in the promoter region (Figure 2a). The triangular connection among PoptrCSE10, -12 and -18 with a high Ks value may be the result of the ancient 'Salicoid' genome duplication and following fission and fusion of chromosomes [30-32]. We can still observe the collinear relationships among AtrCSE7, PoptrCSE12 and PoptrCSE18 as well as the loss of genes in gene strings on the homologous segments (Figure 2c). The duplication block containing PoptrCSE10 is homologous to that with PoptrCSE18, indicating that the duplication event occurred between PoptrCSE10 and PoptrCSE18.

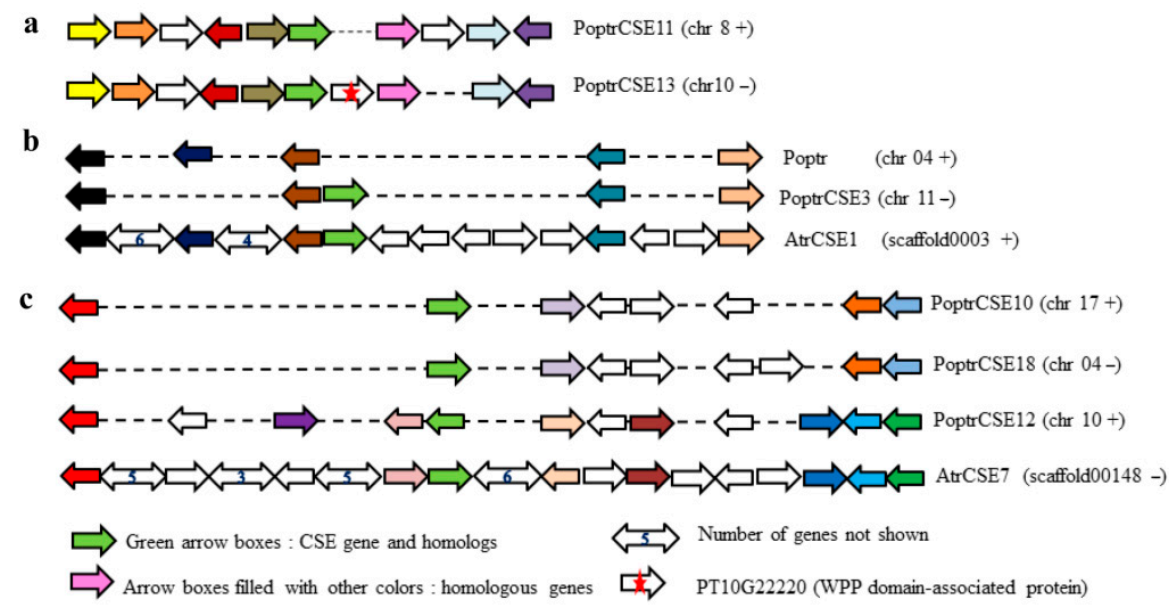

Figure 2. Collinear analysis of homologous segments between Populus and Amborella. The collinear analysis of (a) PoptrCSE11 and PoptrCSE13, (b) PoptrCSE3 and AtrCSE1 and (c) AtrCSE7, PoptrCSE10, PoptrCSE12 and PoptrCSE18. Green arrow boxes present the CSES and CSE homologs; arrow boxes filled with other colors indicate other homologous genes (orthologs or paralogs). The symbols of CSE genes and the chromosomes on which they are located are marked on the right. In particular, Poptr (chr $04+)$ means no CSE homologs were found on this homologous segment (positive strand) on Populus chromosome 04.

\subsection{Promoter Sequence Analysis}

All of the upstream 1500 bp sequences of PoptrCSE and PoptrCSE-like genes were obtained, except for PoptrCSE13, which only has $361 \mathrm{bp}$ to its closest gene. Responsive elements of the six types of hormones were found in different PoptrCSE genes, especially PoptrCSE17, which had elements responding to the six hormones (Table 1) [33-38]. These hormones are important for plant development and defense. AC-rich elements corresponding to the MYB transcription factor-binding motif are necessary for vascular expression [39,40]. PoptrCSE1, with the highest similarity to AtCSE1, had both the AC-I and AC-II elements in its promoter region. PoptrCSE2 and PoptrCSE12 had the AC-I element, and PoptrCSE5 and -6 had the AC-II element. In addition, motifs binding to different MYBs were found in many PoptrCSE genes. Among these PoptrCSE genes, PoptrCSE18 had two MYB-binding motifs, MBS and MBSI, involved in drought response and flavonoid biosynthetic gene regulation, respectively. Some members of the MYB family have been reported as lignin-specific transcription factors [41-44]. In addition, we identified a secondary wall NAC binding element (SNBE) and a secondary wall MYB responsive element (SMRE) in the promoter regions of PoptrCSE and PoptrCSE-like genes, except for PoptrCSE13 (Supplementary Table S2). Both SNBE and SMRE were reported to be involved in secondary cell wall biosynthesis $[45,46]$. Furthermore, cis-elements involved in the stress response were revealed and were widely detected in the PoptrCSE2 and PoptrCSE12 promoter regions. 


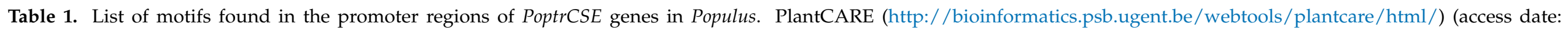

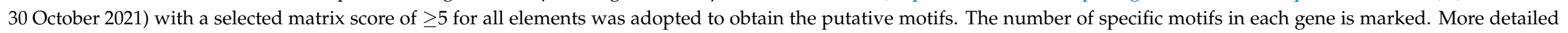

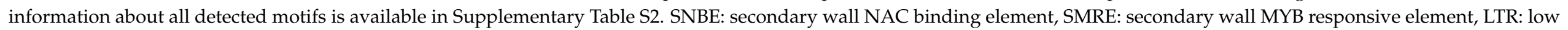
temperature responsive element, HSE: heat stress responsive element.

\begin{tabular}{|c|c|c|c|c|c|c|c|c|c|c|c|c|c|c|c|}
\hline & $\begin{array}{l}\text { Number } \\
\text { of } \\
\text { SNBEs }\end{array}$ & $\begin{array}{l}\text { Number } \\
\text { of } \\
\text { SMREs }\end{array}$ & $\begin{array}{l}\text { Salicylic } \\
\text { Acid }\end{array}$ & MeJA & Gibberellin & Auxin & $\begin{array}{l}\text { Abscisic } \\
\text { Acid }\end{array}$ & Ethylene & $\begin{array}{c}\text { AC-I } \\
\text { Element }\end{array}$ & $\begin{array}{c}\text { AC-II } \\
\text { Element }\end{array}$ & LTR & HSE & $\begin{array}{l}\text { Anaerobic } \\
\text { Induction }\end{array}$ & $\begin{array}{l}\text { Defense and } \\
\text { Stress (TC-Rich } \\
\text { Repeats) }\end{array}$ & $\begin{array}{c}\text { MYB Involved in } \\
\text { Drought } \\
\text { Inducibility (MBS) }\end{array}$ \\
\hline PoptrCSE1 & 4 & 5 & - & - & 1 & - & 1 & - & 2 & 1 & 1 & - & 2 & - & - \\
\hline PoptrCSE2 & 4 & 6 & - & - & - & - & 1 & - & 1 & - & 3 & 3 & - & 4 & - \\
\hline PoptrCSE3 & 1 & 4 & 1 & - & 1 & 1 & - & 1 & - & - & 2 & 2 & 5 & 1 & - \\
\hline PoptrCSE4 & 4 & 1 & - & - & - & - & 1 & - & - & - & - & - & 2 & 1 & 1 \\
\hline PoptrCSE5 & 6 & 2 & - & - & 1 & 1 & - & - & - & 1 & - & - & 2 & 1 & 2 \\
\hline PoptrCSE6 & 4 & 2 & 2 & - & 1 & 1 & 1 & 1 & - & 1 & - & 2 & 3 & 1 & 2 \\
\hline PoptrCSE7 & 3 & 5 & 2 & 1 & - & - & 2 & - & - & - & - & 4 & 1 & 2 & 1 \\
\hline PoptrCSE8 & 5 & 3 & - & 1 & 1 & - & 1 & 2 & - & - & - & - & 2 & - & 1 \\
\hline PoptrCSE9 & 3 & 3 & 1 & - & 1 & 1 & - & 1 & - & - & 2 & 2 & 5 & 1 & - \\
\hline PoptrCSE10 & 2 & 5 & 1 & 2 & - & 1 & 1 & 1 & - & - & - & - & 3 & - & - \\
\hline PoptrCSE11 & 2 & 3 & - & - & - & - & 1 & - & - & - & - & - & 5 & 2 & 1 \\
\hline PoptrCSE12 & 5 & 2 & 1 & 1 & - & - & 1 & 1 & 1 & - & - & 3 & 1 & 1 & 4 \\
\hline PoptrCSE13 & 0 & 0 & - & - & - & - & 1 & - & - & - & 1 & 1 & - & - & - \\
\hline PoptrCSE14 & 7 & 2 & 1 & 2 & 1 & 1 & 1 & - & - & - & - & 3 & 1 & 2 & 3 \\
\hline PoptrCSE15 & 5 & 4 & 1 & 3 & - & 2 & 1 & - & - & - & 1 & 1 & - & 1 & 2 \\
\hline PoptrCSE16 & 5 & 4 & 1 & 3 & - & - & - & - & - & - & - & 2 & 1 & 1 & 2 \\
\hline PoptrCSE17 & 2 & 2 & 1 & 2 & 2 & 1 & 3 & 1 & - & - & 1 & 1 & - & 3 & 4 \\
\hline PoptrCSE18 & 2 & 4 & 1 & 1 & 1 & - & - & - & - & 1 & 3 & 1 & 4 & 2 & 2 \\
\hline
\end{tabular}




\subsection{Alignment and Phylogenetic Analysis of CSE and CSE-like Genes}

Detailed analyses of putative protein sequences of PoptrCSE and AtCSE1 were also performed (Figure 3). The acyltransferase $\left(\mathrm{HX}_{4} \mathrm{D}\right)$ and two hydrolase (GXSXG) motifs were identified in these putative proteins [24,47]. Two GXSXG motifs were highly conserved in all PoptrCSE proteins. $\mathrm{HX}_{4} \mathrm{D}$, which was reported as a key motif for acyltransferase activity for monoacylglycerol acyltransferase (MGAT) in peanuts, was less conserved [47]. While aspartate and glutamate residues in the sixth position were present in most PoptrCSE proteins, this position was substituted by threonine in PoptrCSE10, -12 and -18. PoptrCSE1, -2 and -3 maintained this position, as with AtCSE1. The catalytic triad (S, D and H), which was reported to be responsible for lipase/esterase activity, was identified as highly conserved in these proteins (black circles) [48]. Another two proteins, PoptrCSE14 and PoptrCSE18, were motif deficient. PoptrCSE14 only had the first hydrolase motif without the other two motifs, and PoptrCSE18 lacked the first hydrolase motif and even appeared to have an incomplete catalytic triad because of its shorter peptide sequence. The above variations indicate a functional change or deficiency in these two proteins.

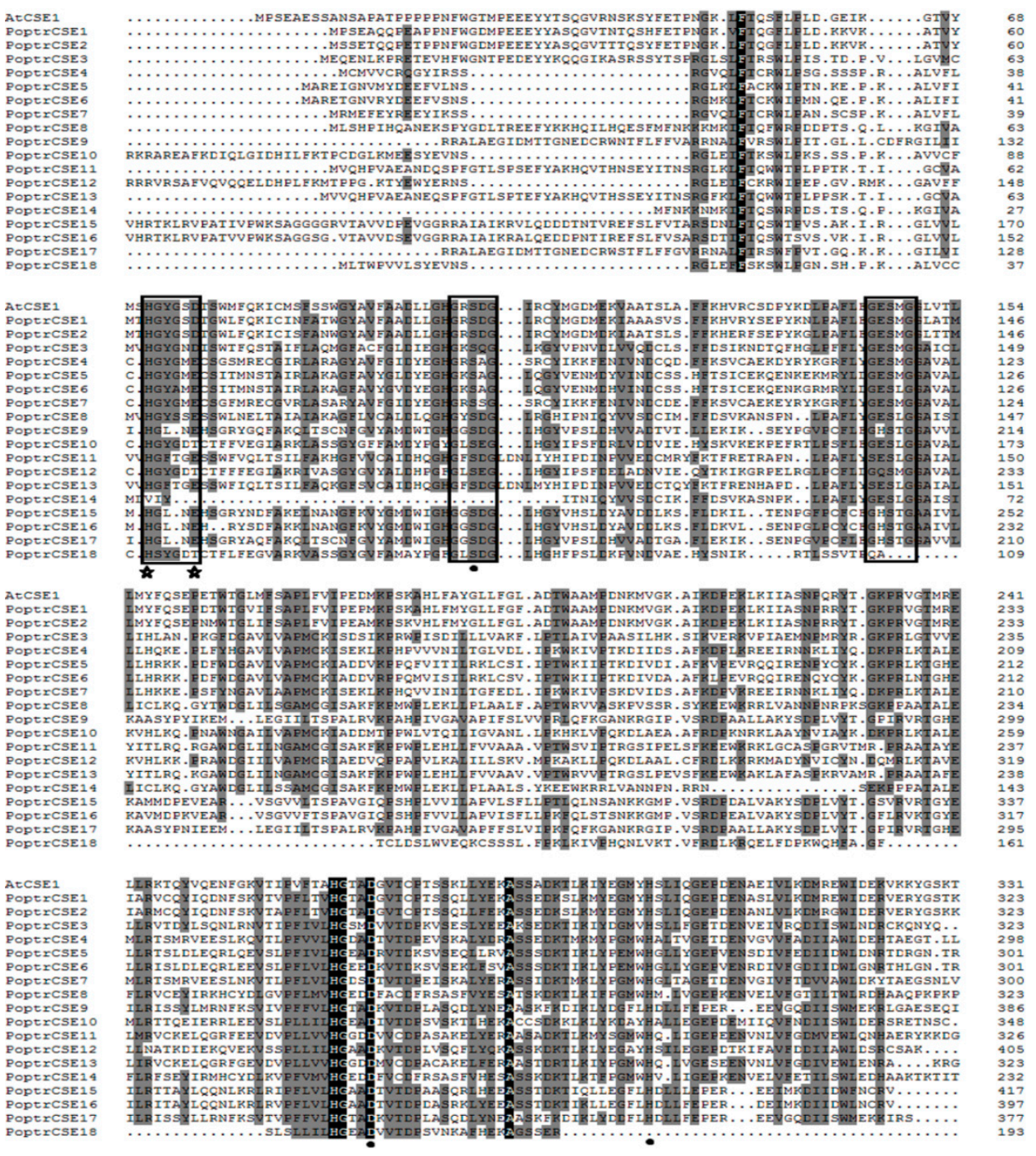

Figure 3. Alignment of eighteen putative PoptrCSE sequences with AtCSE1 (At1g52760). Conserved and functional motifs are indicated in black boxes, including an acyltransferase motif $\left(\mathrm{HX}_{4} \mathrm{D}\right)$ and two lipase/hydrolase motifs (GXSXG). Key amino acids involved in enzymatic activity are also marked with black stars (acyltransferase activity) or circles (catalytic triad). 
Phylogenetic analysis was conducted on a total of 153 putative CSE and CSE-like proteins including AtMAGLs (monoacylglycerol lipase) from 18 species. The hydrolase (GXSXG) motifs in these putative proteins were highly conserved, corresponding to the lipase/esterase superfamily, whereas the $\mathrm{HX}_{4} \mathrm{D}$ motifs experienced divergence, especially in the $\mathrm{D}$ position, which can be used to reveal the evolutionary relationships between these CSEs. Based on the evolution of the acyltransferase motif and the maximum likelihood (ML) bootstrap phylogenetic tree (Figure 4), all of these putative CSE or CSE-like proteins were divided into five groups. It is noteworthy that most of the CSE or CSE-like proteins in Group I have the classical acyltransferase motif with $\mathrm{HX}_{4} \mathrm{D}$. Further analysis indicated that this group could be divided into two subsets. Herein, Group Ia is classified as a bona fide CSE group based on the rules described by Raes et al. (2003) [49]. Putative proteins in this subgroup have high sequence identity with AtCSE1, which was identified as a bona fide CSE in the functional analyses [18]. PoptrCSE1 and -2, bona fide PoptrCSEs, were grouped with AtCSE1 in Group Ia and were likely involved in lignin biosynthesis with CSE activity. It is interesting that bona fide CSEs, except two proteins from Oryza sativa (OsCSE5 and OSINDICA_CSE7), are found primarily in dicots. CSEs in Group Ib are relatively more closely related to bona fide CSEs. Almost all of the putative proteins in Group II and Group III have $\mathrm{HX}_{4} \mathrm{E}$ instead of $\mathrm{HX}_{4} \mathrm{D}$. Group IV refers to CSEs with $\mathrm{HX}_{4} \mathrm{~T} / \mathrm{S}$. The last group (Group V) contains PoptrCSE15, $-16,-17$ and -9 and mainly has $\mathrm{HX}_{4} \mathrm{H}$ instead of the acyltransferase motif. In addition, we conducted a separate phylogenetic analysis of PoptrCSE and PoptrCSE-like proteins with AtMAGLs. The phylogenetic analysis also showed that these PoptrCSE-like proteins were clustered with AtMAGLs (Figure S2). PoptrCSE3 (Group Ib) showed a close relationship with AtMAGL1 which has MAG lipase activities and lysophosphatidylcholine (LPC) and/or lysophosphatidylethanolamine (LPE) hydrolase activities. The catalytic residues of putative CSEs are presented in Supplementary Table S3, grouped according to the phylogenetic tree.

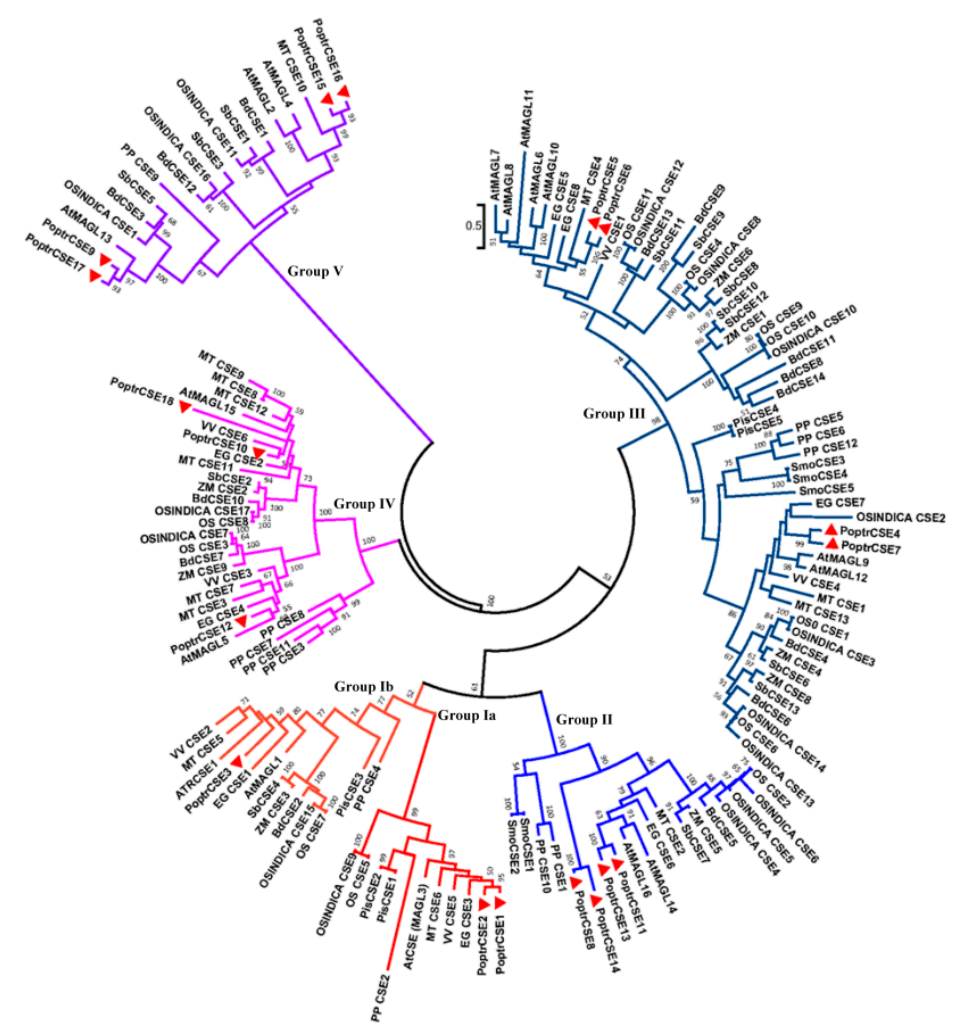

Figure 4. Phylogenetic tree of screened CSE and CSE-like protein sequences from Populus and other species. Eighteen PoptrCSE or PoptrCSE-like proteins are marked with red triangles. All 153 CSE and CSE-like proteins including AtMAGLs are listed in Supplementary Table S5. 


\subsection{Expression Analysis of CSE Genes}

From the results of the hierarchical cluster analysis based on expression levels in different tissues or organs, three expression clusters were found (Figure 5a). PoptrCSE4, -7 and -10 showed obvious expression preferences in mature leaves and clustered with PoptrCSE5 and -13 in cluster I, with a relatively higher expression abundance in mature leaves (ML). PoptrCSE and PoptrCSE-like genes in cluster II had relatively high expression levels in the shoot and leaf primordium (SLp), with no obvious preferences in all detected tissues and organs. PoptrCSE1, $-2,-9,-12$ and -17 , constituting cluster III, showed expression preferences in developing phloem and xylem, which are involved in wood formation. The co-expression network showed that PoptrCSE1 and -2 had a significant correlation with genes involved in lignin biosynthesis, which can be used as evidence to explore the putative function of these PoptrCSEs (Figure 5b).
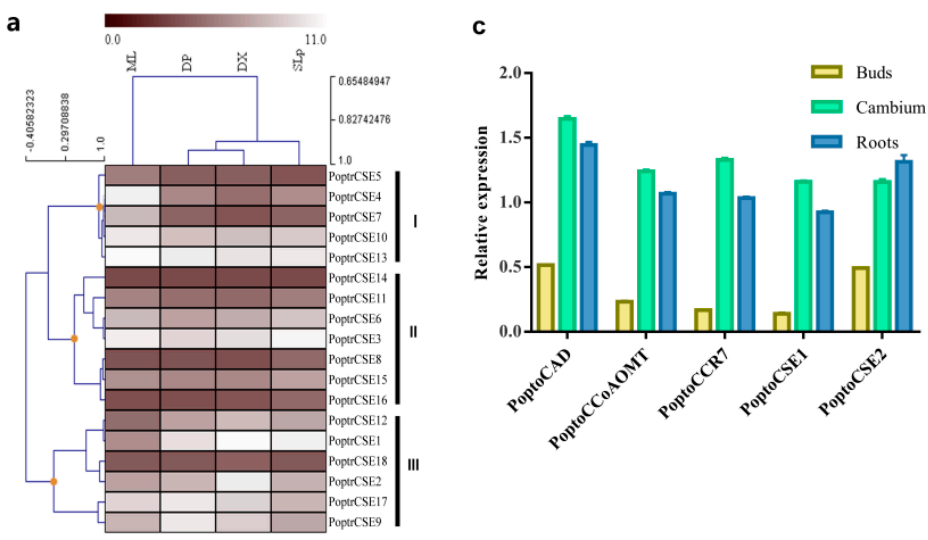

b

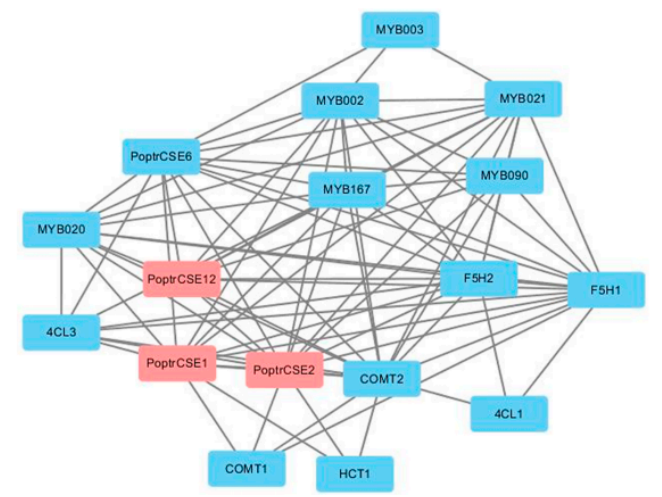

Figure 5. Expression profile and co-expression network of CSE and CSE-like genes in poplar. (a) Expression profile of PoptrCSE and PoptrCSE-like genes in various Populus tissues. Cluster analysis associated with the expression profile is also shown in this figure. Transcript levels are indicated by shading from dark red to white, as shown in the legend. Tissues or specific parts of plants are indicated with the respective abbreviations: DP, developing phloem; DX, developing xylem; ML, mature leaf; SLp, shoot and leaf primordium. The support information including the log2-transformed signal intensity and statistical test is available in Supplementary Table S6. (b) Co-expression network of PoptrCSE and PoptrCSE-like genes with identified genes involved in lignin biosynthesis. PoptrCSE1, -2 and -12 are marked with red boxes. (c) Similar expression pattern of PoptoCSE1 and -2, PoptoCAD, PoptoCCR7 and PoptoCCoAOMT using semi-qRT-PCR.

\subsection{Characteristics of PoptoCSE1 and PoptoCSE2}

We further cloned and obtained the corresponding PoptoCSE1, -2 and -12 from $P$. tomentosa. PoptoCSE1, -2 and -12 have high sequence identity $(>98 \%)$ with the corresponding CSEs in P. trichocarpa. Semi-qRT-PCR (semi-quantitative reverse transcription polymerase chain reaction) showed that PoptoCSE1 and PoptoCSE2 had a similar expression 
pattern to other monolignol biosynthesis-related genes such as PoptoCAD, PoptoCCR7 and PoptoCCoAOMT (caffeoyl-CoA 3-O-methyltransferas) (Figure 5c). Both PoptoCSE1 and PoptoCSE2 with fused GFP (green fluorescent protein) were observed in the plasma membrane and ER, and the signal became clearer after plasmolysis (Figure 6). This result corresponds with the subcellular localization of AtCSE (At1g52760), which was reported as LysoPL2 (Lysophospholipase 2) [24].

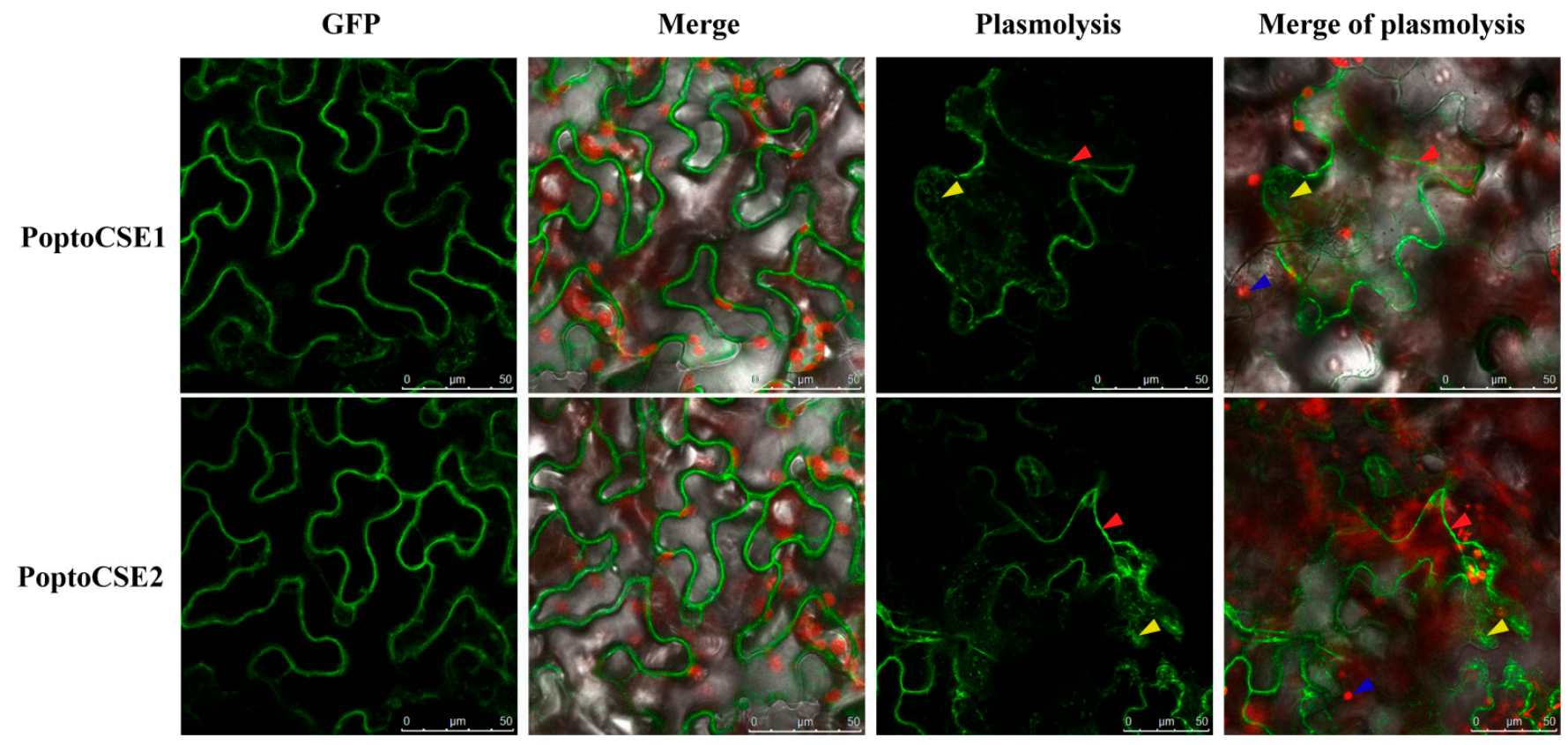

Figure 6. Subcellular localization of PoptoCSE1 and PoptoCSE2 in transformed tobacco leaves. The red arrow, yellow arrow and blue arrow indicate the plasma membrane, ER and chloroplast, respectively.

\subsection{Enzymatic Assay of PoptoCSE1, -2 and -12 of P. tomentosa}

The recombinant proteins PoptoCSE1 and PoptoCSE2 were obtained and purified for enzymatic assay. We detected the CSE activity using HPLC-MS and found that PoptoCSE1 and PoptoCSE2 have detectable activity against caffeoyl shikimate (Figure 7a-c), while PoptoCSE12 showed no activity against caffeoyl shikimate (Figure S1). In addition, we also explored the optimum reaction temperature and $\mathrm{pH}$ for PoptoCSE1 and PoptoCSE2 (Figure 7d,e). PoptoCSE1 and PoptoCSE2 have a similar temperature profile and pH profile (except at pH 8.0-9.0) for catalyzing caffeoyl shikimate, and the highest activities for PoptoCSEs were obtained at $\mathrm{pH}=7.0$ and a temperature of $40{ }^{\circ} \mathrm{C}$. PoptoCSE1 and PoptoCSE2 showed similar biochemical properties against caffeoyl shikimate. 

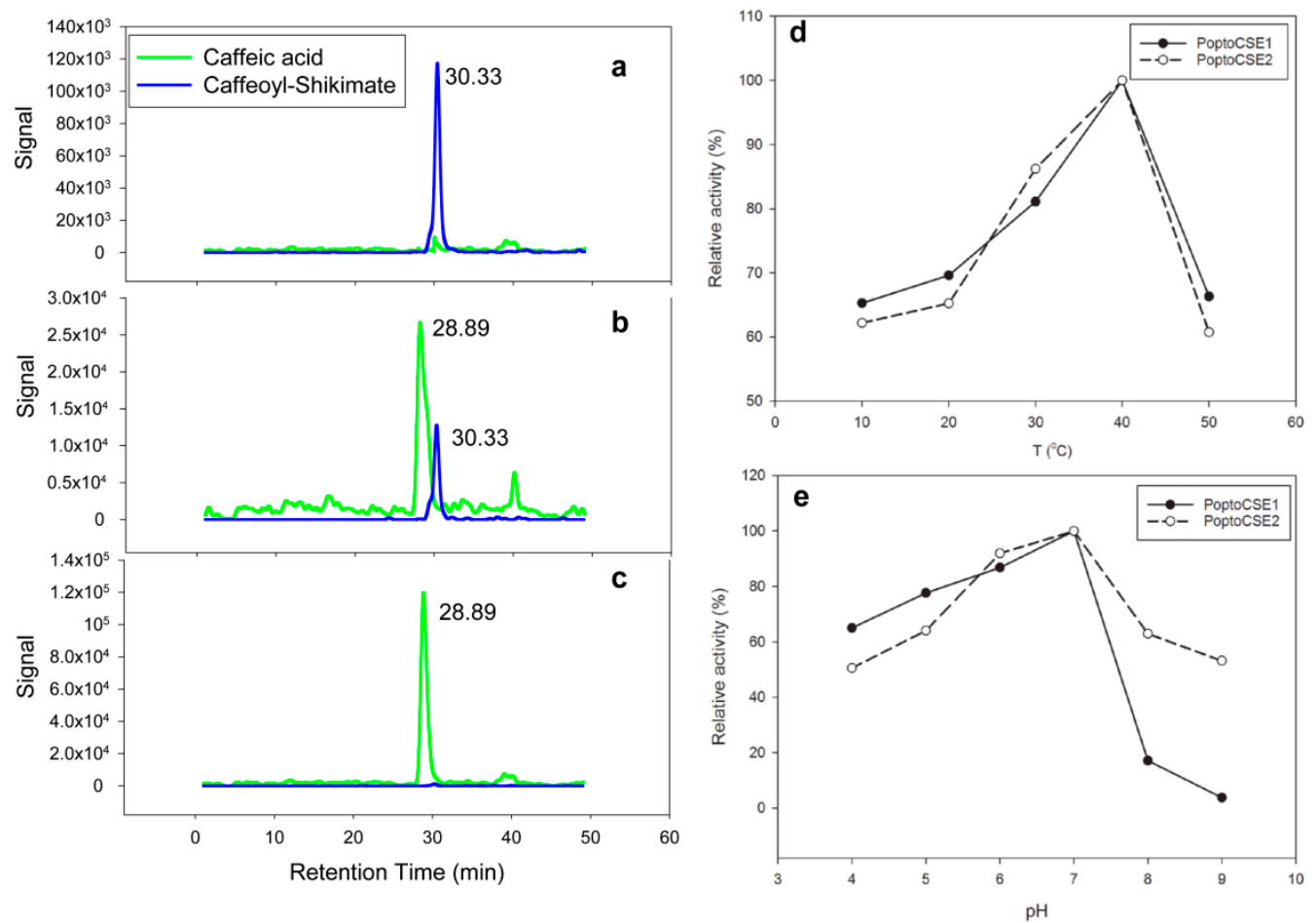

Figure 7. Enzymatic assay of PoptoCSE1 and PoptoCSE2. (a-c) The decrease in caffeoyl shikimate and the increase in caffeic acid along with the reaction process catalyzed by PoptoCSE1 with different reaction times: $5 \mathrm{~s} \mathrm{(a),} 80 \mathrm{~s}$ (b) and $120 \mathrm{~s}$ (c). $(\mathbf{d}, \mathbf{e})$ The temperature $(\mathbf{d})$ and $\mathrm{pH}(\mathbf{e})$ profiles for recombinant PoptoCSE1 and PoptoCSE2 activities. The mean value of three replicates was used to calculate the relative activity.

\section{Discussion}

Phylogenetic analysis and comparison of the acyltransferase motif of 153 putative CSEs suggested the existence of 5 CSE and CSE-like groups. PoptrCSE1 and PoptrCSE2 were identified as bona fide CSEs in class I. Additionally, we reported a genuine CSE in the gymnosperm Larix kaempferi [27]. The deficiency of CSE in most monocots corresponds to findings of studies in B. distachyon and Z. may [26]. It is possible that the ancestral CSE appears before the divergence of angiosperms and gymnosperms. The bona fide CSEs (Group Ia) were likely lost in most monocots at the divergence between dicots and monocots. AtCSE was first annotated as LysoPL2 and also identified as a member of the MAGL gene family [24,50]. Here, we further classified these CSE-like proteins into five groups with different amino acid replacements at the last position of the acyltransferase motif. Phylogenetic analysis also showed that these PoptrCSE-like proteins are clustered with AtMAGLs (Figures 4 and S2).

Based on the above analysis in the present study, we primarily proposed functions of PoptrCSE and PoptrCSE-like genes. PoptrCSE1, -2 and -12 are expressed mainly in developing xylem (cluster III). Co-expression analysis showed that PoptrCSE1, -2 and -12 had a significant correlation with genes involved in lignin biosynthesis. The cis-elements AC-I, AC-II, SMREs and SNBEs are involved in regulating secondary cell wall biosynthesis $[41,46]$. It has been reported that MYB58 or MYB63 can bind the AC element, the conserved promoter sequence in most monolignol biosynthetic genes, to directly activate the expression of these genes in A. thaliana [51]. PoptrCSE1, -2 and -12 all contain the AC element (AC-I or AC-II) in the promoter regions. SMREs, additional cis-elements along with to the AC elements and Arabidopsis MYB46/83, are master switches of the entire secondary wall biosynthetic program and are able to bind to SMREs [52]. Secondary wall NACs (SWNs), as master transcriptional switches activating secondary wall biosynthesis, were reported to bind to SNREs. The SNBE sites binding with SWNs were proposed to be necessary for a combinatorial activation of the secondary wall biosynthesis genes 
with other transcription factors [53]. In our promoter analysis, SMREs and SNREs were found to widely distribute in most CSE and CSE-like gene promoter regions, and Pop$\operatorname{trCSE} 1$ and -2 contained the most SMREs and SNBEs (Table 1). PoptrCSE1 and -2 have high sequence identity with AtCSE1, both of which have many cis-elements involved in secondary wall biosynthesis. The promoter analysis revealed that PoptrCSE2 and -12 had many cis-elements involved in stress response that differ from PoptrCSE1. This indicates that PoptrCSE1, -2 and -12 are likely involved in wood formation, but PoptrCSE2 and PoptrCSE12 might respond mainly to stress. This functional divergence has been reported in other key genes involved in lignin biosynthesis [54-56].

In addition, PoptrCSE3, belonging to phylogenetic Group Ib, showed a close relationship with AtMAGL1 which was reported to harbor MAG lipase activities and LPC and/or LPE hydrolase activities. PoptrCSE3 and AtMAGL1 have both acyltransferase $\left(\mathrm{HX}_{4} \mathrm{D}\right)$ and hydrolase (GXSXG) motifs and are proposed to have acyltransferase activity. PoptrCSE6, -7 , $-9,-14,-15,-16$ and -17 from phylogenetic groups III and $\mathrm{V}$ all have cis-elements involved in response to hormones. Previous studies have revealed the biofunction of AtCSE and its function in phospholipid metabolism [24,47]. PoptrCSE9, -15, -16 and -17 clustered with AtMAGL2 and -4 which were reported to have MAG lipase activities and LPC and/or LPE hydrolase activities. In addition, expression of AtMAGL2 and -4 was significantly induced by salt, osmotic and cold stresses. The roles of lipases in jasmonate metabolism and phosphatidic acid signaling associated with plant defense have also been reported [57]. Therefore, CSE-like proteins in this class likely function as lipases and are thought to be involved in plant defense in response to salicylic acid or MeJA. Other PoptrCSE-like genes also have analogous AtMAGLs and might also have MAG lipase activity.

\section{Materials and Methods}

\subsection{Plant Materials}

Buds, cambial zone tissue and roots of six-year-old P. tomentosa 741 were harvested as plant material in this research from Hebei, China. The cambial zone tissue was collected by cutting the bark longitudinally with a knife, then removing a large piece of the bark and using a scalpel to scrape the cambial zone tissue from the outer layer of the xylem and the inner layer of the phloem. Collected samples were immediately frozen in liquid nitrogen and then stored at $-80^{\circ} \mathrm{C}$ until use. Three biological replicates were performed for each sample.

\subsection{Genome-Wide Identification of CSE Gene Family Members}

To identify the CSE sequences in Populus, we first performed a BlastP search in the NCBI database using AtCSE1 as a query [58]. Sequences from selected species with high scores, query cover $>90 \%$, identity $>50 \%$ and a low E-value $\left(1 \times 10^{-100}\right)$ were collected to build an HMM model. An HMM search was used to find more sequences with the same features using the hidden Markov model, avoiding missing sequences based on alignment. The results of the HMM search were further screened based on scores and E-values $\left(<1 \times 10^{-5}\right)$ of both the domain and full sequence. Finally, the screening sequences were submitted to the Superfamily 1.75 database (https:/ / supfam.org/SUPERFAMILY/) (access date: 9 December 2021) to further determine their superfamily and family in the SCOP database. Details are available in Supplementary Table S4. The CSE sequences used for phylogenetic analysis (Supplementary Table S5) were screened from various sources based on the platform PLAZA 3.0 (http://bioinformatics.psb.ugent.be/plaza/) (access date: 8 January 2021). CSE sequences from the gymnosperm $P$. sitchensis and the fern $S$. moellendorffii were obtained using BlastP in the NCBI database. The rules proposed by Raes et al. (2003) to distinguish bona fide genes and 'likes' were used to determine bona fide CSE and CSE-like genes [49]. 


\subsection{Organization and Distribution of CSE Genes and Homologs on Populus Chromosomes}

The identified CSE sequences in Populus were submitted to GSDS 2.0 server (http:/ / gsds.gao-lab.org/) (access date: 7 December 2021) to visualize the intron-exon organization patterns of these genes. Then, CSE and CSE homologs were located on their respective chromosomes in specific duplicated blocks based on the Populus genome. Duplicated blocks were determined using WGDotplot in the PLAZA platform. A schematic diagram was constructed to describe the organization and distribution of CSE and its homologs on Populus chromosomes.

\subsection{Promoter Analysis of PoptrCSE Genes}

Promoter sequences of PoptrCSE genes were extracted using the PLAZA platform with the parameter upstream $1500 \mathrm{bp}$. Promoter analysis was performed by querying all CSE genes for the upstream or up to the closest gene using the PlantCARE database (http: // bioinformatics.psb.ugent.be/webtools/plantcare/html/) (access date: 30 October 2021) with a selected matrix score of $\geq 5$ for all elements. SNBE and SMRE were detected based on the motif sequences reported by Zhong et al. (2012) [52]. Details are presented in Supplementary Table S2.

\subsection{CSE Sequence Alignment and Phylogenetic Analysis}

Both DNAman 8.0 (Lynnon BioSoft) and CLUSTAL W software assembled in MEGA 5.05 software were used to align candidate PoptrCSEs and bona fide CSEs using default parameters [59]. A phylogenetic tree was obtained using Mega 5.05 with the maximum likelihood method, the JTT substitution model and a model with G + I rates among sites. The reliability of internal branches was assessed by bootstrapping using 1000 bootstrap replicates and marked above nodes only if greater than 50 . The putative CSE protein sequences used are listed in Supplementary Table S5.

\subsection{RNA Extraction and cDNA Synthesis}

Total RNA from different tissues was extracted using Plant RN38 Kit (Aidlab, Beijing, China) according to the manual. The quality of RNA was evaluated by both Nanodrop one (Thermo Scientific, Waltham, MA, USA) and electrophoresis. cDNA was synthesized with the PC54-TRUEscript RT kit (Aidlab, Beijing, China) according to the manufacturer's protocol.

\subsection{CSE Expression Profile in P. trichocarpa and P. tomentosa}

Gene expression profiling for various tissues including DP, DX, ML and SLp in P. trichocarpa was performed using the GEO database with the accession number GSE30507. Transcriptome datasets of these samples were re-analyzed by submitting them to Pop's Pipes: Poplar Gene Expression Pipelines (http:/ / sys.bio.mtu.edu/) (access date: 7 December 2021). In addition, the RNA-seq dataset GSE78953 was also used for confirming CSE gene expression, and co-expression profiling based on the Pearson correlation coefficient with genes involved in lignin biosynthesis was also performed for exploring the putative function of PoptrCSE and PoptrCSE-like genes. GSE78953 contains the transcriptome information of xylem tissue of transgenic and wild-type P.trichocarpa. MultiExperiment Viewer (MeV) version 4.9 and Cytoscape 3.4 were used to visualize the CSE expression profile or coexpression network. The detailed information is available in Supplementary Table S6. Semi-qRT-PCR was performed for PoptoCSE1, PoptoCSE2 and several other monolignol biosynthesis genes in $P$. tomentosa. Actin was used as a reference gene.

\subsection{Subcellular Localization of PoptoCSE}

The methods used for the subcellular localization of PoptoCSE and plasmolysis were in accordance with our previous study [27]. CSEs (PoptoCSE1,-2 and -12) were cloned from the cambial zone tissue in $P$. tomentosa based on the sequence information from $P$. trichocarpa. The primer pairs and PCR procedure are listed in Supplementary Table S7. The pBI121 
vector with PoptoCSE1 and PoptoCSE2 and GFP fusion expression was constructed by restriction enzymes and then ligation with T4 ligase (Takara, Dalian, China). The recombinant plasmids pBI121-35S-PoptoCSE1 and pBI121-35S-PoptoCSE2 were transferred into Agrobacterium tumefaciens strain GV3101, which was transferred into tobacco leaves via Agrobacterium-mediated transient transformation [60]. The tobacco leaves were cut into squares of $5 \mathrm{~mm} \times 5 \mathrm{~mm}$, and GFP fluorescence in leaves was observed using a Leica TCS SP8 confocal microscope (Leica Microsystems, Wetzlar, Germany).

\subsection{Purification of Recombinant CSE and HCT}

HCT can catalyze caffeoyl-CoA and shikimic acid to produce caffeoyl shikimate which is the substrate of CSEs. To obtain caffeoyl shikimate, we cloned HCT from the cambial zone tissue in P. tomentosa. The CSEs and HCT6 were constructed with pET28a (Novagen) and then transformed into E. coli BL21 (DE3). The recombinant proteins tagged with His were purified using Ni-NTA agarose (Qiagen). After washing the column using lysis buffer with gradient concentrations of imidazole, the His-tagged PoptoCSEs and PoptoHCT were eluted with $100 \mathrm{mM}$ imidazole in lysis buffer [27]. The eluted proteins were detected by SDS-PAGE and stained by Coomassie Brilliant Blue R250. The concentration of the purified protein was determined using Bradford Protein Assay Kit (Sangon Biotech, Shanghai, China).

\subsection{Enzymatic Assay of Recombinant PoptoCSEs}

We obtained caffeoyl shikimate using enzymatic reaction catalyzed by purified recombinant PoptoHCT6 with caffeoyl-CoA and shikimic acid. The reaction was performed according to the methods provided by Escamilla-Treviño et al. (2014) [61]. Caffeoyl-CoA was chemically synthesized and authenticated as reported in our previous study [62]. Purified PoptoCSEs were incubated at $30^{\circ} \mathrm{C}$ for 30 min with $100 \mathrm{mM} \mathrm{NaPO}_{4}$ buffer (pH 7.5), $500 \mu \mathrm{M}$ dithiothreitol and $100 \mu \mathrm{M}$ caffeoyl shikimate in a final volume of $100 \mu \mathrm{L}[18,23,26]$. The control reactions were also performed with proteins that had been boiled for $10 \mathrm{~min}$. The reactions (including controls) were terminated by adding $10 \mu \mathrm{L}$ of glacial acetic acid. CSE activity was monitored by the appearance of caffeic acid. To explore the optimum reaction temperature, the reactions were incubated at various temperatures that ranged from 10 to $50{ }^{\circ} \mathrm{C}$. Multi-pH phosphate buffers ranging from 4 to 9 were adopted for exploring the optimum reaction $\mathrm{pH}$ for CSE. Reaction products with $100 \mathrm{ng}$ sinapic acids which were added as an internal standard were further purified using microporous filters, then injected into an HPLC with a reverse-phase C18 column (ZORBAX 300SB-C18, $2.1 \times 150 \mathrm{~mm}, 3.5 \mu \mathrm{m}$; Agilent, Santa Clara, CA, USA) and separated in a step gradient using $1 \%$ formic acid in water as solvent $\mathrm{A}$ and acetonitrile as solvent $\mathrm{B}$. The gradient profile was $100 \% \mathrm{~A}$, decreased to $65 \% \mathrm{~A}$ in $24 \mathrm{~min}$, then to $0 \% \mathrm{~A}$ in $3 \mathrm{~min}$ and maintained for $5 \mathrm{~min}$ and then increased to $100 \% \mathrm{~A}$ in $3 \mathrm{~min}$ and maintained for $20 \mathrm{~min}$. The flow rate was $0.15 \mathrm{~mL} / \mathrm{min}$. The substrates and products were identified using an ion trap mass spectrometer (LCQ DECA XP MAX) coupled with an ESI source (Thermo Finnigan). The parameters used for MS analysis were sheath gas (nitrogen) flow rate, $40 \mathrm{arb}$; aux/sweep gas (nitrogen) flow rate, $10 \mathrm{arb}$; spray voltage, $4.5 \mathrm{keV}$; capillary temperature, $320^{\circ} \mathrm{C}$. The MS raw data are available in Supplementary Table S8.

\subsection{GenBank Accession Number}

The Genbank accession numbers of PtoHCT6 and PtoCSE1, -2 and -12 are KT020989, KT020990, KT020997 and KT021003, respectively.

\subsection{Statistical Analyses}

The correlation analysis and ANOVA analysis were performed using SPSS19.0. Significance was marked at $p<0.05$. 


\section{Conclusions}

In this study, we conducted a systematic analysis of the CSE gene family in poplar and studied the possible role of CSE and CSE-like genes in poplar. PoptrCSE1 and PoptrCSE2 were identified as bona fide CSEs via phylogenetic analysis. Co-expression analysis showed that PoptrCSE1, -2 and -12 are significantly related to genes involved in lignin biosynthesis. Additionally, most of the PoptrCSE-like genes are homologs of the AtMAGL gene in Arabidopsis and may act as MAG lipase in poplar. We identified two bona fide CSEs, PoptrCSE1 and PoptrCSE2, by enzymatic assays in vitro and found that PoptoCSE1 and PoptoCSE2 had detectable activity on caffeoyl shikimate, and similar tissue expression patterns and subcellular localization. All these results provide more evidence that two bona fide CSEs are indeed widespread and function in monolignol biosynthesis in Populus.

Supplementary Materials: The following are available online at https:/ /www.mdpi.com/article/10.3 390/ijms222413366/s1.

Author Contributions: Y.G. conceived and designed the research. X.W., N.C., A.Z. and J.K. conducted experiments and analyzed data. X.W. and N.C. wrote the manuscript. X.J. and Y.G. checked the article and provided advice. All authors have read and agreed to the published version of the manuscript.

Funding: This research was funded by the National Natural Science Foundation (NSF 31300498 to Y.G.) and the Beijing Higher Education Young Elite Teacher Project (YETP0755 granted to YING GAI).

Institutional Review Board Statement: Not applicable.

Informed Consent Statement: Not applicable.

Data Availability Statement: The data presented in this study are available in the article and Supplementary Materials.

Acknowledgments: We thank Qi Qi from the College of Horticulture of China Agriculture University for her assistance with the enzymatic assay.

Conflicts of Interest: The authors declare no conflict of interest.

$\begin{array}{ll}\text { Abbreviations } & \\ 4 C L & \text { 4-coumarate: CoA ligase } \\ \text { C3'H } & \text { P-coumarate 3-hydroxylase } \\ \text { C4H } & \text { Cinnamate 4-hydroxylase } \\ \text { CAD } & \text { Cinnamyl alcohol dehydrogenase } \\ \text { CCoAOMT } & \text { Caffeoyl-CoA 3-O-methyltransferas } \\ \text { CCR } & \text { Cinnamoyl-CoA reductase } \\ \text { DP } & \text { Developing phloem } \\ \text { DX } & \text { Developing xylem } \\ \text { ER } & \text { Endoplasmic reticulum } \\ \text { F5H } & \text { Ferulate 5-hydroxylase } \\ \text { GFP } & \text { Green fluorescent protein } \\ \text { HCT } & \text { Hydroxylcinnamoyl-CoA shikimate/quinate hydroxycinnamoyl transferase } \\ \text { HSE } & \text { Heat stress responsive element } \\ \text { LTR } & \text { Low temperature responsive element } \\ \text { LysoPL2 } & \text { Lysophospholipase 2 } \\ \text { MAGL } & \text { Monoacylglycerol lipase } \\ \text { MGAT } & \text { Monoacylglycerol acyltransferase } \\ \text { ML } & \text { Mature leaf } \\ \text { PAL } & \text { Phenylalanine ammonia-lyase } \\ \text { Semi-qRT-PCR } & \text { Semi-quantitative reverse transcription polymerase chain reaction } \\ \text { SLp } & \text { Shoot and leaf primordium } \\ \text { SMRE } & \text { Secondary wall MYB responsive element } \\ \text { SNBE } & \text { Secondary wall NAC binding element } \\ \end{array}$




\section{References}

1. Poovaiah, C.R.; Nageswara-Rao, M.; Soneji, J.R.; Baxter, H.L.; Stewart, C.N., Jr. Altered lignin biosynthesis using biotechnology to improve lignocellulosic biofuel feedstocks. Plant Biotechnol. J. 2014, 12, 1163-1173. [CrossRef]

2. Vanholme, R.; Demedts, B.; Morreel, K.; Ralph, J.; Boerjan, W. Lignin biosynthesis and structure. Plant Physiol. 2010, 153, 895-905. [CrossRef] [PubMed]

3. Bhuiyan, N.H.; Selvaraj, G.; Wei, Y.; King, J. Gene expression profiling and silencing reveal that monolignol biosynthesis plays a critical role in penetration defence in wheat against powdery mildew invasion. J. Exp. Bot. 2009, 60, 509-521. [CrossRef]

4. Wang, J.P.; Matthews, M.L.; Williams, C.M.; Shi, R.; Yang, C.; Tunlaya-Anukit, S.; Chen, H.-C.; Li, Q.; Liu, J.; Lin, C.-Y.; et al. Improving wood properties for wood utilization through multi-omics integration in lignin biosynthesis. Nat. Commun. 2018, 9, 1579. [CrossRef] [PubMed]

5. Ragauskas, A.J.; Beckham, G.T.; Biddy, M.J.; Chandra, R.; Chen, F.; Davis, M.F.; Davison, B.H.; Dixon, R.A.; Gilna, P.; Keller, M.; et al. Lignin valorization: Improving lignin processing in the biorefinery. Science 2014, 344, 1246843. [CrossRef] [PubMed]

6. Zhao, Q.; Dixon, R.A. Altering the cell wall and its impact on plant disease: From forage to bioenergy. Annu. Rev. Phytopathol. 2014, 52, 69-91. [CrossRef]

7. Tang, W.; Tang, A.Y. Transgenic woody plants for biofuel. J. For. Res. 2014, 25, 225-236. [CrossRef]

8. Vanholme, R.; Storme, V.; Vanholme, B.; Sundin, L.; Christensen, J.H.; Goeminne, G.; Halpin, C.; Rohde, A.; Morreel, K.; Boerjan, W. A systems biology view of responses to lignin biosynthesis perturbations in Arabidopsis. Plant Cell 2012, 24, 3506-3529. [CrossRef] [PubMed]

9. Li, X.; Chapple, C. Understanding lignification: Challenges beyond monolignol biosynthesis. Plant Physiol. 2010, 154, 449-452. [CrossRef] [PubMed]

10. Voelker, S.L.; Lachenbruch, B.; Meinzer, F.C.; Strauss, S.H. Reduced wood stiffness and strength, and altered stem form, in young antisense 4CL transgenic poplars with reduced lignin contents. New Phytol. 2011, 189, 1096-1109. [CrossRef]

11. Stewart, J.; Akiyama, T.; Chapple, C.; Ralph, J.; Mansfield, S. The effects on lignin structure of overexpression of ferulate 5-hydroxylase in hybrid poplar. Plant Physiol. 2009, 150, 621-635. [CrossRef] [PubMed]

12. Punith, N.; Wang, J.P.; Ronald, S.; Vincent, C.; Cranos, W.; Ducoste, J.J.; Daniel, C. Assessing the impact of the 4CL enzyme complex on the robustness of monolignol biosynthesis using metabolic pathway analysis. PLoS ONE 2018, 13, e0193896. [CrossRef]

13. Vanholme, R.; Meester, B.D.; Ralph, J.; Boerjan, W. Lignin biosynthesis and its integration into metabolism. Curr. Opin. Biotechnol. 2019, 56, 230-239. [CrossRef]

14. Qiao, Z. Lignification: Flexibility, biosynthesis and regulation. Trends Plant Sci. 2016, 21, 713-721. [CrossRef]

15. Whetten, R.; Sederoff, R. Lignin biosynthesis. Plant Cell 1995, 7, 1001-1013. [CrossRef]

16. Boerjan, W.; Ralph, J.; Baucher, M. Lignin biosynthesis. Annu. Rev. Plant Biol. 2003, 54, 519-546. [CrossRef]

17. Weng, J.K.; Chapple, C. The origin and evolution of lignin biosynthesis. New Phytol. 2010, 187, 273-285. [CrossRef]

18. Vanholme, R.; Cesarino, I.; Rataj, K.; Xiao, Y.; Sundin, L.; Goeminne, G.; Kim, H.; Cross, J.; Morreel, K.; Araujo, P.; et al. Caffeoyl shikimate esterase (CSE) is an enzyme in the lignin biosynthetic pathway in Arabidopsis. Science 2013, 341, 1103-1106. [CrossRef]

19. Wang, J.; Matthews, M.; Naik, P.; Williams, C.; Ducoste, J.; Sederoff, R.; Chiang, V. Flux modeling for monolignol biosynthesis. Curr. Opin. Biotechnol. 2019, 56, 187-192. [CrossRef]

20. Guo, L.; Wang, P.; Jaini, R.; Dudareva, N.; Chapple, C.; Morgan, J. Dynamic modeling of subcellular phenylpropanoid metabolism in Arabidopsis lignifying cells. Metab. Eng. 2018, 49, 36-46. [CrossRef]

21. Faraji, M.; Fonseca, L.; Escamilla-Treviño, L.; Barros-Rios, J.; Engle, N.; Yang, Z.; Tschaplinski, T.; Dixon, R.; Voit, E. Mathematical models of lignin biosynthesis. Biotechnol. Biofuels 2018, 11, 34. [CrossRef]

22. Faraji, M.; Fonseca, L.; Escamilla-Treviño, L.; Barros-Rios, J.; Engle, N.; Yang, Z.; Tschaplinski, T.; Dixon, R.; Voit, E. A dynamic model of lignin biosynthesis in Brachypodium distachyon. Biotechnol. Biofuels 2018, 11, 253. [CrossRef]

23. Saleme, M.L.S.; Cesarino, I.; Vargas, L.; Kim, H.; Vanholme, R.; Goeminne, G.; Van Acker, R.; Fonseca, F.C.A.; Pallidis, A.; Voorend, W.; et al. Silencing caffeoyl shikimate esterase affects lignification and improves saccharification in poplar. Plant Physiol. 2017, 175, 1040-1057. [CrossRef]

24. Gao, W.; Li, H.Y.; Xiao, S.; Chye, M.L. Acyl-CoA-binding protein 2 binds lysophospholipase 2 and lysoPC to promote tolerance to cadmium-induced oxidative stress in transgenic Arabidopsis. Plant J. Cell Mol. Biol. 2010, 62, 989-1003. [CrossRef] [PubMed]

25. Wang, J.P.; Naik, P.P.; Chen, H.C.; Shi, R.; Lin, C.Y.; Liu, J.; Shuford, C.M.; Li, Q.; Sun, Y.H.; Tunlaya-Anukit, S.; et al. Complete proteomic-based enzyme reaction and inhibition kinetics reveal how monolignol biosynthetic enzyme families affect metabolic flux and lignin in Populus trichocarpa. Plant Cell 2014, 26, 894-914. [CrossRef] [PubMed]

26. Ha, C.M.; Escamilla-Trevino, L.; Yarce, J.C.; Kim, H.; Ralph, J.; Chen, F.; Dixon, R.A. An essential role of caffeoyl shikimate esterase in monolignol biosynthesis in Medicago truncatula. Plant J. Cell Mol. Biol. 2016, 86, 363-375. [CrossRef]

27. Wang, X.; Chao, N.; Zhang, M.; Jiang, X.; Gai, Y. Functional characteristics of caffeoyl shikimate esterase in Larix kaempferi and monolignol biosynthesis in gymnosperms. Int. J. Mol. Sci. 2019, 20, 6071. [CrossRef]

28. de Vries, L.; Brouckaert, M.; Chanoca, A.; Kim, H.; Regner, M.R.; Timokhin, V.I.; Sun, Y.; De Meester, B.; Van Doorsselaere, J.; Goeminne, G.; et al. CRISPR-Cas9 editing of caffeoyl shikimate esterase 1 and 2 shows their importance and partial redundancy in lignification in Populus tremula $\times$ P. alba. Plant Biotechnol. J. 2021, 19, 2221-2234. [CrossRef] 
29. Jang, H.A.; Bae, E.K.; Kim, M.H.; Park, S.J.; Choi, N.Y.; Pyo, S.W.; Lee, C.; Jeong, H.Y.; Lee, H.; Choi, Y.I.; et al. CRISPR-Knockout of CSE Gene improves saccharification efficiency by reducing lignin content in hybrid poplar. Int. J. Mol. Sci. 2021, $22,9750$. [CrossRef] [PubMed]

30. Tuskan, G.A.; Difazio, S.; Jansson, S.; Bohlmann, J.; Grigoriev, I.; Hellsten, U.; Putnam, N.; Ralph, S.; Rombauts, S.; Salamov, A.; et al. The genome of black cottonwood, Populus trichocarpa (Torr. \& Gray). Science 2006, 313, 1596-1604. [CrossRef]

31. Dai, X.; Hu, Q.; Cai, Q.; Feng, K.; Ye, N.; Tuskan, G.; Milne, R.; Chen, Y.; Wan, Z.; Wang, Z.; et al. The willow genome and divergent evolution from poplar after the common genome duplication. Cell Res. 2014, 24, 1274-1277. [CrossRef] [PubMed]

32. Hou, J.; Ye, N.; Dong, Z.; Lu, M.; Li, L.; Yin, T. Major Chromosomal rearrangements distinguish willow and poplar after the ancestral "Salicoid" genome duplication. Genome Biol. Evol. 2016, 8, 1868-1875. [CrossRef] [PubMed]

33. Song, J.; Wang, Z. Molecular cloning, expression and characterization of a phenylalanine ammonia-lyase gene (SmPAL1) from Salvia miltiorrhiza. Mol. Biol. Rep. 2008, 36, 939-952. [CrossRef] [PubMed]

34. Ogawa, M.; Hanada, A.; Yamauchi, Y.; Kuwahara, A.; Kamiya, Y.; Yamaguchi, S. Gibberellin biosynthesis and response during Arabidopsis seed germination. Plant Cell 2003, 15, 1591-1604. [CrossRef] [PubMed]

35. Hobo, T.; Asada, M.; Kowyama, Y.; Hattori, T. ACGT-containing abscisic acid response element (ABRE) and coupling element 3 (CE3) are functionally equivalent. Plant J. Cell Mol. Biol. 1999, 19, 679-689. [CrossRef] [PubMed]

36. Hao, D.; Ohme-Takagi, M.; Sarai, A. Unique mode of GCC box recognition by the DNA-binding domain of ethylene-responsive element-binding factor (ERF domain) in plant. J. Biol. Chem. 1998, 273, 26857-26861. [CrossRef] [PubMed]

37. Shah, J.; Klessig, D. Identification of a salicylic acid-responsive element in the promoter of the tobacco pathogenesis-related ß-1,3-glucanase gene, PR-2d. Plant J. Cell Mol. Biol. 1997, 10, 1089-1101. [CrossRef]

38. Guilfoyle, T.; Hagen, G.; Li, Y.; Ulmasov, T.; Liu, Z.B.; Strabala, T.; Gee, M. Auxin-regulated transcription. Aust. J. Plant Physiol. 1993, 20, 489-502. [CrossRef]

39. Hartmann, U.; Sagasser, M.; Mehrtens, F.; Stracke, R.; Weisshaar, B. Differential combinatorial interactions of cis-acting elements recognized by R2R3-MYB, BZIP, and BHLH factors control light-responsive and tissue-specific activation of phenylpropanoid biosynthesis genes. Plant Mol. Biol. 2005, 57, 155-171. [CrossRef]

40. Hatton, D.; Sablowski, R.; Yung, M.H.; Smith, C.; Schuch, W.; Bevan, M. Two classes of cis sequences contribute to tissue-specific expression of a PAL2 promoter in transgenic tobacco. Plant J. Cell Mol. Biol. 1995, 7, 859-876. [CrossRef]

41. Zhao, Q.; Dixon, R.A. Transcriptional networks for lignin biosynthesis: More complex than we thought? Trends Plant Sci. 2011, 16, 227-233. [CrossRef]

42. Legay, S.; Sivadon, P.; Blervacq, A.S.; Pavy, N.; Baghdady, A.; Tremblay, L.; Levasseur, C.; Ladouce, N.; Lapierre, C.; Seguin, A.; et al. EgMYB1, an R2R3 MYB transcription factor from eucalyptus negatively regulates secondary cell wall formation in Arabidopsis and poplar. New Phytol. 2010, 188, 774-786. [CrossRef]

43. Öhman, D.; Demedts, B.; Kumar, M.; Gerber, L.; Gorzsás, A.; Goeminne, G.; Hedenström, M.; Ellis, B.; Boerjan, W.; Sundberg, B. MYB103 is required for ferulate-5-hydroxylase expression and syringyl lignin biosynthesis in Arabidopsis stems. Plant J. Cell Mol. Biol. 2012, 73, 63-76. [CrossRef] [PubMed]

44. Fornale, S.; Sonbol, F.-M.; Maes, T.; Capellades, M.; Puigdomènech, P.; Rigau, J.; Caparros-Ruiz, D. Down-regulation of the maize and Arabidopsis thaliana caffeic acid o-methyl-transferase genes by two new maize R2R3-MYB transcription factors. Plant Mol. Biol. 2007, 62, 809-823. [CrossRef] [PubMed]

45. McCarthy, R.; Zhong, R.; Yu, L. Secondary wall NAC binding element (SNBE), a key cis-acting element required for target gene activation by secondary wall NAC master switches. Plant Signal. Behav. 2011, 6, 1282-1285. [CrossRef] [PubMed]

46. Zhong, R.; Ye, Z.H. Complexity of the transcriptional network controlling secondary wall biosynthesis. Plant Sci. Int. J. Exp. Plant Biol. 2014, 229, 193-207. [CrossRef]

47. Vijayaraj, P.; Jashal, C.B.; Vijayakumar, A.; Rani, S.H.; Venkata Rao, D.K.; Rajasekharan, R. A bifunctional enzyme that has both monoacylglycerol acyltransferase and acyl hydrolase activities. Plant Physiol. 2012, 160, 667-683. [CrossRef] [PubMed]

48. Wang, A.J.; Loo, R.; Chen, Z.L.; Dennis, E. Regiospecificity and catalytic triad of lysophospholipase I. J. Biol. Chem. 1997, 272, 22030-22036. [CrossRef]

49. Raes, J.; Rohde, A.; Christensen, J.H.; Van de Peer, Y.; Boerjan, W. Genome-wide characterization of the lignification toolbox in Arabidopsis. Plant Physiol. 2003, 133, 1051-1071. [CrossRef]

50. Kim, R.J.; Kim, H.J.; Shim, D.; Suh, M.C. Molecular and biochemical characterizations of the monoacylglycerol lipase gene family of Arabidopsis thaliana. Plant J. 2016, 85, 758-771. [CrossRef]

51. Zhou, J.; Lee, C.; Zhong, R.; Ye, Z.H. MYB58 and MYB63 are transcriptional activators of the lignin biosynthetic pathway during secondary cell wall formation in Arabidopsis. Plant Cell 2009, 21, 248-266. [CrossRef] [PubMed]

52. Zhong, R.; Ye, Z.H. MYB46 and MYB83 bind to the SMRE sites and directly activate a suite of transcription factors and secondary wall biosynthetic genes. Plant Cell Physiol. 2012, 53, 368-380. [CrossRef]

53. Zhong, R.; Lee, C.; Ye, Z.H. Global analysis of direct targets of secondary wall NAC master switches in Arabidopsis. Mol. Plant 2010, 3, 1087-1103. [CrossRef]

54. Tronchet, M.; Balague, C.; Kroj, T.; Jouanin, L.; Roby, D. Cinnamyl alcohol dehydrogenases-C and D, key enzymes in lignin biosynthesis, play an essential role in disease resistance in Arabidopsis. Mol. Plant Pathol. 2010, 11, 83-92. [CrossRef]

55. Moura, J.C.; Bonine, C.A.; de Oliveira Fernandes Viana, J.; Dornelas, M.C.; Mazzafera, P. Abiotic and biotic stresses and changes in the lignin content and composition in plants. J. Integr. Plant Biol. 2010, 52, 360-376. [CrossRef] 
56. Lauvergeat, V.; Lacomme, C.; Lacombe, E.; Lasserre, E.; Roby, D.; Grima-Pettenati, J. Two cinnamoyl-CoA reductase (CCR) genes from Arabidopsis thaliana are differentially expressed during development and in response to infection with pathogenic bacteria. Phytochemistry 2001, 57, 1187-1195. [CrossRef]

57. Shah, J. Lipases in signaling plant defense responses. In Signaling and Communication in Plants; Wang, X., Ed.; Phospholipases in plant signaling; Springer: Berlin, Germany, 2014; Volume 20, pp. 207-228.

58. Coordinators, N.R. Database resources of the national center for biotechnology information. Nucleic Acids Res. 2017, 46, D8-D13. [CrossRef]

59. Tamura, K.; Peterson, D.; Peterson, N.; Stecher, G.; Nei, M.; Kumar, S. MEGA5: Molecular evolutionary genetics analysis using maximum likelihood, evolutionary distance, and maximum parsimony methods. Mol. Biol. Evol. 2011, 28, 2731-2739. [CrossRef] [PubMed]

60. Morita, S.; Yamashita, Y.; Fujiki, M.; Todaka, R.; Nishikawa, Y.; Hosoki, A.; Yabe, C.; Nakamura, J.I.; Kawamura, K.; Suwastika, I.; et al. Expression of a rice glutaredoxin in aleurone layers of developing and mature seeds: Subcellular localization and possible functions in antioxidant defense. Planta 2015, 242, 1195-1206. [CrossRef]

61. Escamilla-Trevino, L.L.; Shen, H.; Hernandez, T.; Yin, Y.; Xu, Y.; Dixon, R.A. Early lignin pathway enzymes and routes to chlorogenic acid in switchgrass (Panicum virgatum L.). Plant Mol. Biol. 2014, 84, 565-576. [CrossRef] [PubMed]

62. Chao, N.; Li, N.; Qi, Q.; Li, S.; Lyu, T.; Jiang, X.; Gai, Y. Characterization of the cinnamoyl-CoA reductase (CCR) gene family in Populus tomentosa reveals the enzymatic active sites and evolution of CCR. Planta 2017, 245, 61-75. [CrossRef] [PubMed] 